\title{
High Performance Redox Flow Batteries: An Analysis of the Upper Performance Limits of Flow Batteries Using Non-aqueous Solvents
}

\author{
C.-N. Sun, ${ }^{a}$ M. M. Mench ${ }^{b}$ and T. A. Zawodzinski ${ }^{a, c}$ \\ ${ }^{a}$ Physical Chemistry of Materials Group, Oak Ridge National Lab, Oak Ridge, TN, USA \\ ${ }^{\mathrm{b}}$ Department of Mechanical, Aerospace, and Biomedical Engineering, University of \\ Tennessee, Knoxville, TN, 37996 \\ ${ }^{c}$ Department of Chemical and Biomolecular Engineering, University of Tennessee, \\ Knoxville, TN 37996-2200, USA
}

Redox Flow Batteries (RFBs) are a promising technology for grid-scale electrochemical energy storage. In this work, we use a recently achieved highperformance flow battery performance curve as a basis to assess the maximum achievable performance of a RFB employing non-aqueous solutions as active materials. First we show high performance in a vanadium redox flow battery (VRFB), specifically a limiting situation in which the cell losses are ohmic in nature and derive from electrolyte conductance. Based on that case, we analyze the analogous limiting behavior of non-aqueous (NA) systems using a series of calculations assuming similar ohmic losses, scaled by the relative electrolyte resistances, with a higher voltage redox couple assumed for the NA battery. The results indicate that the NA battery performance is limited by the low electrolyte conductivity to a fraction of the performance of the VRFB. Given the narrow window in which the NARFB offers advantages, even for the most generous limiting assumptions related to performance while ignoring the numerous other disadvantageous aspects of these systems, we conclude that this technology is unlikely under present circumstances to provide practical large-scale energy storage solutions. 


\section{Introduction}

Large-scale electrochemical energy storage is enjoying a surge of interest world-wide as an important component of systems for sustainably obtaining electricity with minimal environmental impact using renewable resources.[1] Solar and wind energy are renewable sources[2, 3] that are, in general, readily available. However, an operational challenge arises because solar and wind sources are intermittent in nature.[4] Electrical energy storage devices can be applied as mediators between renewable energy farms and the electric grid, smoothing out the intermittency and enabling grid-level power generation for average, rather than peak, requirements.

Among the available energy storage technologies for large-scale applications, redox flow batteries (RFBs), as illustrated in Figure 1, are promising candidates for this application.[5] Unlike conventional batteries, RFBs store redox active components separately from the electrochemical 'conversion' apparatus and therefore decouple the energy and the power, allowing relatively easy and inexpensive scale-up as well as improved safety features, as discussed in the various reviews of the technology.[such as 6, 7, 8, 9] In RFB systems, the volume and concentration of the solution determine the amount of energy that can be stored. On the other hand, the intrinsic performance of the cell controls the power density of the system and thus the size of the electrochemical converters. RFBs, in general, can be categorized in terms of the type of electrolyte used into aqueous systems,[10-13] non-aqueous systems,[14-16] and hybrid systems.[17] The latter include systems in which some of the redox states might be present in a solid state, such as a metal, in which case one electrode stores energy in the charged state. Several distinct characteristics among the proposed systems are the operating voltage window, the range of working temperature, cost, and safety.

Since the cell performance is strongly related to the cost of the entire system, $[18,19]$ significant efforts have been made to enhance performance of the aqueous systems.[2022] In this work, we show representative data for a high performance all-vanadium redox flow battery (VRFB), with a peak power density of $1340 \mathrm{~mW} / \mathrm{cm}^{2}$. The polarization curve of this cell exhibits essentially a straight line, suggesting that only ohmic processes dominate the voltage loss during operation. This is a limiting, best-case scenario in that 
other sources of loss are minimized. We note that the operating conditions for this experiment included use of a small $\left(5 \mathrm{~cm}^{2}\right)$ cell with a simple serpentine flow field and high flow rates. Testing in such a cell minimizes effects of flow or maldistribution of current, issues that are more effectively probed in larger-scale and more practical cells. However, with the elimination of those factors, such testing conditions provide a fine test bed for materials properties and their effects on cell performance. This is the primary emphasis in the analysis to come. By carrying out testing in this cell, we can also obtain meaningful results from polarization curve analysis. We can use conditions of very low utilization to avoid state-of-charge effects, as we have discussed elsewhere. When translated to a larger-scale system level, these results are subject to many other considerations, including balance of plant efficiency, notably the pumping power associated with high flow rate, and of course the cell does not operate in a short-length polarization mode in a real system.

Non-aqueous redox flow batteries (NARFB) have been proposed by several researchers as a means to improve the energy density of such systems, with the extended voltage window of the potential redox couples as a major advantage.[14, 15, 23] Nevertheless, the voltage loss due to ohmic effects is likely to be more significant in NARFBs, given that the conductivity of non-aqueous organic solvent-based electrolyte is usually at least two orders of magnitude lower than that of the aqueous acidic media. We note that the term non-aqueous refers to a very broad class of electrolytes and the conductivity of such systems as ionic liquids and similar solvents can be higher. In most ILs to date, however, the transference of the electro-active species is not necessarily high. This motivates us to compare the best-case performance limit for the aqueous and the non-aqueous RFBs by estimating the ohmic loss of the systems.

In general, the losses in the cell stem from thermodynamic, kinetic and mass transport limitations in addition to ohmic losses related to electrolyte and membrane conductivity. Thermodynamic losses show up primarily in concentration polarization effects in the cell; kinetic losses stem from any sluggish kinetics at the positive or negative electrodes; mass transport losses occur within the active porous electrode. In our previously published work, we have analyzed kinetics of electrode processes and indicated that these indeed can be sluggish. However, we also have shown that in a cell employing a suitable 
electrode with high internal surface areas, there is little to no loss incurred from electrode kinetics even with a carbon electrode material. Mass transport limitations will be discussed in more detail below. Considering the high-performance VRFB data to represent a proof-of concept that we can indeed achieve pure ohmic limitation in such a device, we calculate best-case performance of non-aqueous systems using a simple ohmic model. Note that several additional factors which can be unfavorable for the NARFBs are not considered in the calculation. These are discussed at the end of this communication. This motivates a discussion of design-limiting aspects for favorable applications of the NARFB.

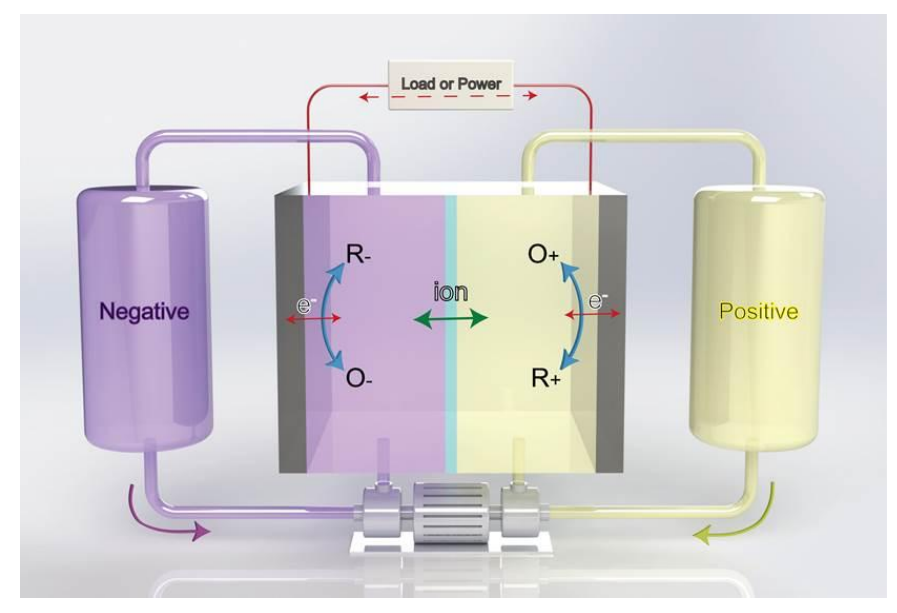

Figure 1. Schematic illustration of the redox flow battery system

Recently, Darling et al. [24] presented an analysis that compared various options, including aqueous and non-aqueous redox flow battery options. However, the level of detail presented regarding performance was limited. Here we present a more detailed analysis of the latter. The point of this paper is a focus on the materials limitations incurred by some different classes of chemistry used. The losses referred to above from systems and from state of charge are likely to be similar for the aqueous and NARFB cases (though we suggest that Darling's analysis is slightly too favorable to NARFBs in terms of cost, at least.) We believe our analysis will stimulate consideration of what cell components limit performance and why, perhaps leading to improvement in NARFBs that we do not take into account in the analysis grounded in present-day reality. 


\section{Experimental}

\section{Vanadium solution}

Vanadium solutions were prepared by dissolving vanadium(IV) sulfate oxide hydrate (99.9\%, Alfa Aesar) into diluted sulfuric acid. $1.7 \mathrm{M} \mathrm{VO}_{2}^{+}$and $1.7 \mathrm{M} \mathrm{V}^{2+}$ in $5 \mathrm{M}$ total sulfate solution were prepared by electrochemically charging the initial solution at $1.8 \mathrm{~V}$ until the charging current density fell to $<10 \mathrm{~mA} / \mathrm{cm}^{2}$ in a nitrogen environment. The resultant volume of each solution was $400 \mathrm{~mL}$.

\section{Cell configuration}

Battery testing was conducted using a zero-gap cell configuration. ${ }^{17}$ As described previously, the $5 \mathrm{~cm}^{2}$ single cell (Fuel Cell Technologies) hardware consisted of aluminum end plates, gold-plated current collectors, and graphite blocks with a single serpentine flow field for electrolyte distribution. Multiple sheets of experimental grade carbon paper, referred as CP-ESA, were stacked at each side and served as the electrode of the battery. A single piece of Nafion 211 (Ion Power) was used as the membrane for the battery.

\section{Polarization curve testing}

Prior to the polarization measurement, the cell was conditioned by sequentially charging and discharging at $200 \mathrm{~mA} / \mathrm{cm}^{2}$ for several $10 \mathrm{~min}$ steps. The discharge polarization curve measurement ${ }^{20}$ was performed using a Bio-Logic potentiostat coupled with a $20 \mathrm{~A}$ booster. An applied voltage was held for 10 seconds to develop a steady state current followed by impedance spectroscopy over the frequency range from $5 \mathrm{kHz}$ to 1 $\mathrm{kHz}$ for measuring high frequency resistance (HFR). The solution in each compartment of the individual electrodes was not recirculated. Instead, the outlet from each electrode was directed to a separate outlet bottle. This operation mode is referred to as "single pass" and allows us to maintain a controlled state of charge at each operating current. A constant flow rate of $90 \mathrm{~mL} / \mathrm{min}$ was maintained with a 2 channel peristaltic pump. The battery and the solution bottles were installed in an environmental chamber set at $30^{\circ} \mathrm{C}$. Each electrolyte storage bottle was blanketed with flow of ultra-high purity nitrogen.

\section{Results and discussion}




\subsection{VRB Pol Curve result and discussion}

A typical discharge polarization curve of the system described above, shown in Figure 2, shows a maximum power density of $1341 \mathrm{~mW} / \mathrm{cm}^{2}$ and a highest ( limiting) current density of $2866 \mathrm{~mA} / \mathrm{cm}^{2}$. This achieved power density is $75 \%$ higher than the one we reported previously.[22] Improved electrode properties along with high concentration electrolyte at high flow rate minimize the losses due to kinetic and electro-active reagent mass transport processes. The cell voltage therefore shows a linear behavior as a function of current density, as seen in Figure 2. This result suggests the performance is dominated by ohmic loss across the entire range of current density. We further correct the cell voltage with areal specific resistance (ASR) measured at high frequency and the associated current density yielding an IR-free voltage. Figure 2 indicates that the IR-free voltage also decreases in a linear fashion as a function of current density. We attribute the remaining loss to the ionic resistance arising from the need to transport ions to and from the reaction site within the electrode.

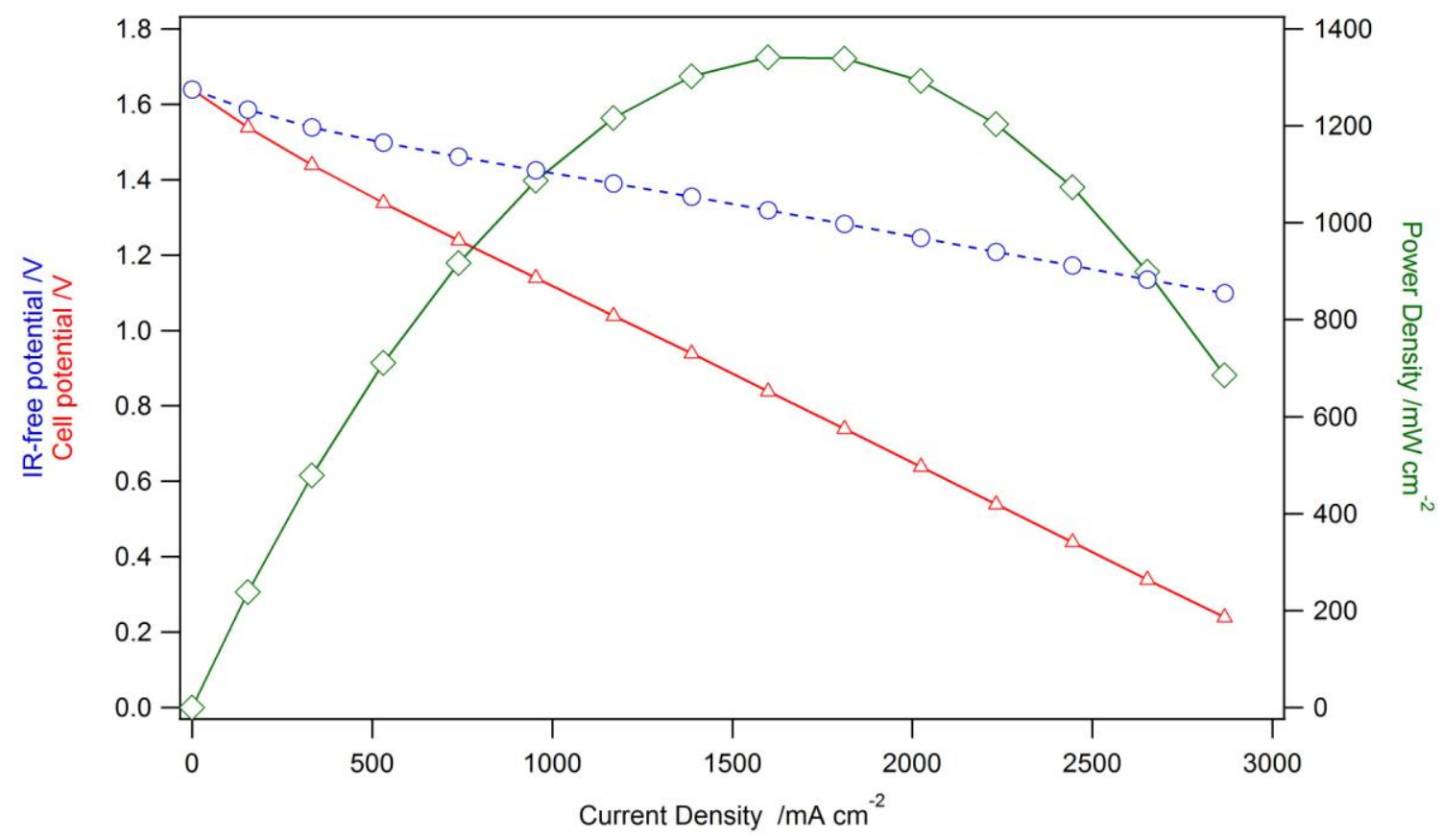


Figure 2. Discharge polarization curve for the vanadium redox flow battery. Overall cell voltage (triangles), IR-free cell voltage (circles), and power density (diamonds) as a function of current density

\section{Calculation Basis}

\section{Assumptions for NARFB}

We now build from the result shown above to compare the redox flow battery performance limit of aqueous and hypothetical non-aqueous systems. Given our ability to achieve the limiting case of all-ohmic loss in the cell, we primarily focus on the ohmic loss element for an idealized non-aqueous system as a "best-case" scenario. The losses attributed to the kinetic process and the diffusion-limited mass transport are therefore ignored in this present work. The ohmic loss estimated by Ohm's law is associated with the ionic conductivity of the materials and the length of the ionic pathways. Again, we emphasize that this is a high-performance limit and, though many aspects of performance of a NARFB (or any aqueous counterpart, for that matter) may fall short of this limit, this will define the maximum achievable performance of the flow battery for given ohmic loss though battery components.

\subsection{Calculation results}

\section{Base Case}

The calculated cell voltage and power density for the NARFB and the VRFB are plotted as a function of current density as shown in Figure 3. Note that the ohmic loss considered in this case is only attributed to the pathway between two electrodes, i.e. within membrane or separator. The parameters used for this 'base case' calculation are listed in Table 1.

\subsubsection{VRFB}

For the VRFB system, the OCV of $1.65 \mathrm{~V}$ was chosen for the high state of charge (SoC) case. The ASR used was obtained from the measured HFR, which includes the contact resistance between each layer, electronic resistance of the carbon sheets and flow field plates, and ionic resistance of the membrane. The membrane, i.e. Nafion 211 in this case, has a dry thickness of $25 \mu \mathrm{m}$. Even for such a thin membrane, the membrane resistance 
constitutes a very large fraction of the observed HFR. We note in passing that this ASR value does not represent an ultimate limit for VRFBs but rather is a conservative value based on the data available at the time of the calculations.

\subsubsection{NARFB}

In the case of the NARFB, an $\mathrm{OCV}$ of $3.5 \mathrm{~V}$ which might be observed for a lithium system[20] was used for the base calculation. This directly introduces the most important performance advantage of the NARFB - the ability to operate from a high initial voltage. The ionic conductivity of $10^{-2} \mathrm{~S} / \mathrm{cm}$ (a relatively high value reported for $1 \mathrm{M} \mathrm{LiPF6}$ in $\mathrm{EC} / \mathrm{DMC}$ at $25^{\circ} \mathrm{C}$ ) of the non-aqueous electrolyte obtained from literature[22] and the MacMullin number of the separator of 8 [26,27] were adopted for ASR estimation using the following equation

$$
A S R=\frac{l}{\sigma} \times N_{m}
$$

where $l$ is the separator thickness, $\sigma$ is the electrolyte conductivity and $N_{m}$ is the Macmullin number.

The ionic resistance in the electrode, contact resistance and electronic resistance of the electrode were also ignored. A Celgard separator of $25 \mu \mathrm{m}$ was used to reflect the standard thickness in current Li battery technologies.[29] The result therefore represents a kinetic and mass transfer loss free, ideal system with only ohmic losses through the separator considered. We note in passing that it is not a 'free lunch' when using thin electrolytes as cross-over of species could have a negative impact on coulombic efficiency that increases with thinner membranes. Nonetheless, our results suggest that this can be managed.

\subsubsection{VRFB vS NARFB}

Figure 3 compares the performance by merely considering the ASR of the two systems. In both cases, the reaction was assumed to take place exactly at the closest part of electrode to the separator, in other words, the ionic resistance in the electrode was not considered. With higher OCV compared to the aqueous system, the non-aqueous system shows better performance at low current density regime in this case. However, the 
operating voltage for the NARFB decreases more drastically as current density increases owing to a higher ASR as compared to VRFB. It should be noted that the ASR used here for the NARFB is 'over-idealized' in that it only considers electrolyte contributions, whereas the ASR for VRFB obtained from the experimental result includes all resistive elements i.e. contact resistance and electronic resistance of the electrode. Nonetheless, in the limit of an essentially 'flat' electrode and very high electrolyte conductivity for the NARFB, the initial behavior appears promising.
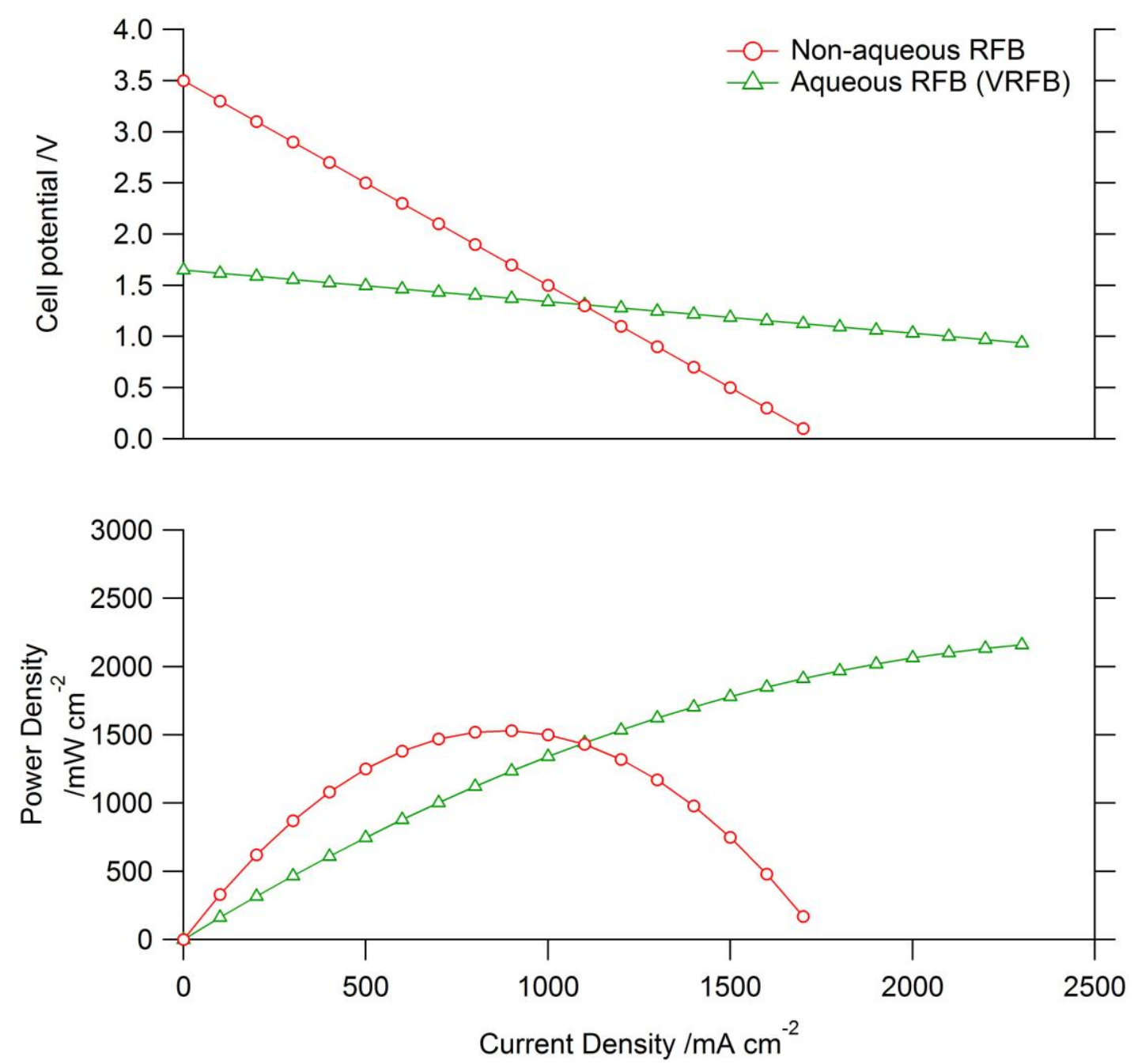

Figure 3. Calculated polarization and power density curves for the vanadium redox flow battery (triangles) and the non-aqueous redox flow battery (circles) as a function of current density.

Table 1. Parameters for the NRFB and VRB performance calculation 


\begin{tabular}{ccccc}
\hline & OC & Conductivity & Electrolyte \\
V $(\mathbf{V}]$ & $(\mathbf{S} / \mathbf{c m}]$ & $\begin{array}{c}\text { MacMu } \\
\text { thickness }(\mu \mathrm{m}]\end{array}$ & $\begin{array}{c}\text { llin } \\
\text { number }\end{array}$ \\
\hline NARFB & 3.5 & $10^{-2}$ & 25 & 8 \\
VRB & 1.65 & 0.021 & 25 & - \\
\hline
\end{tabular}

\subsubsection{ASR dependence}

For the NARFB, the electrolyte conductivity varies with adopted materials. This depends on the salt and solvent used. Optimized non-aqueous liquid electrolytes have the conductivities ranging from $10^{-2}$ to $10^{-3} \mathrm{~S} / \mathrm{cm}$.[26] The conductivities of solid electrolytes such as polymer electrolytes, [28-30] glass-ceramics developed by Ohara Inc.,[31] and Lipon[32] are within the range from $10^{-3}$ to $10^{-6} \mathrm{~S} / \mathrm{cm}$. However, the latter are not available in a mechanically stable form for a large format battery such as a typical gridscale RFB. Solid electrolytes, perhaps, can be processed into a film thinner than the separator to reduce the ASR, but here we compare various conductivities at the same thickness.

Figure 4 shows the calculated polarization curves for the NARFB based on the parameters given in Table 1 except the electrolyte conductivities vary from $10^{-2}$ to $10^{-4}$ $\mathrm{S} / \mathrm{cm}$. Again, Figure 4 only considers the ionic conductivity through the separator. As expected, the decrease of electrolyte conductivity induces significant performance decrease. The power density for the $10^{-3} \mathrm{~S} / \mathrm{cm}$ case becomes lower than that for the VRFB as current density above $100 \mathrm{~mA} / \mathrm{cm}^{2}$. In addition, OCV of the system can vary based on the adopted chemistry.[14, 15, 33-41] Based on the parameters in Table 1, Figure 5 shows the performance variation as OCV changes. If we define the operating efficiency as the ratio of operating voltage to $\mathrm{OCV}$, the change of electrolyte conductivity and OCV would strongly affect the current available at given operating efficiency. 

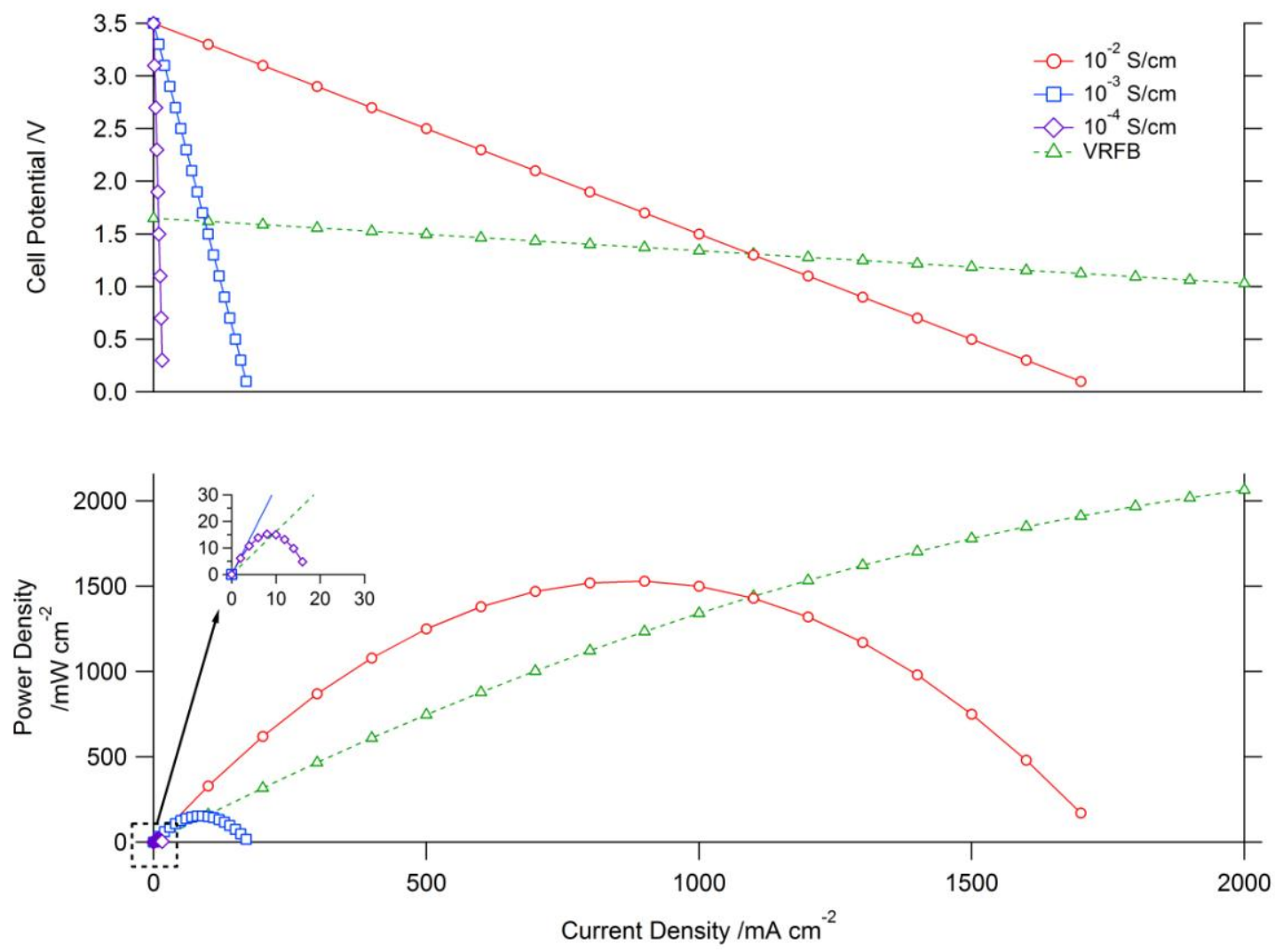

Figure 4. Calculated polarization and power density curves for the NARFB with electrolyte conductivity $10^{-2}$ (circles), $10^{-3}$ (squares), and $10^{-4}$ (diamonds) $\mathrm{S} / \mathrm{cm}$ as a function of current density. The result from VRFB is included (triangles) for comparison. 

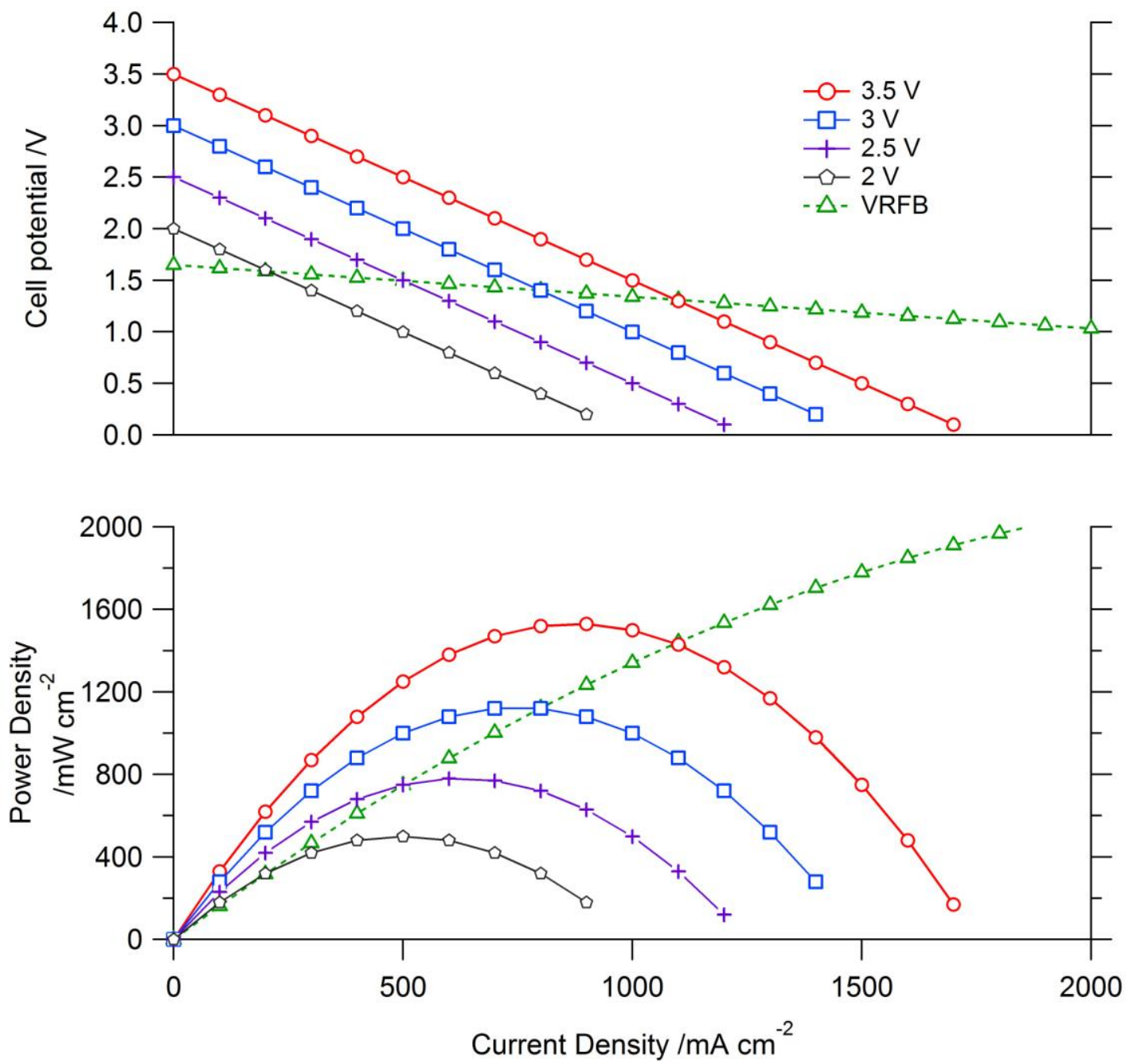

Figure 5 Calculated polarization and power density curves for the NARFB with OCV 3.5 (circles), 3 (squares), 2.5 (crosses) and 2 (stars) volt as a function of current density. The result from VRFB is included (triangles) for comparison.

\subsubsection{Effect of ionic resistance within the electrode}

The slope of the IR-free curve shown in Figure 2 represents the ASR for ionic conduction in the electrode. With the benefit of the constant OCV VRFB system given by the single pass mode, we are able to estimate the length of ionic pathway in the electrode according to Equation [1]. The calculated path length is $1.38 \mathrm{~mm}$, which represents the total conduction length for both electrodes including the tortuosity factor. If we assume that the loss due to the ionic conduction in the electrode is symmetric for positive and negative electrode, the conduction length for each side would be $690 \mu \mathrm{m}$. The thickness of 
the electrode (t) is approximately $1.6 \mathrm{~mm}$ in the cell with $20 \%$ compression. This result suggests that the reaction zone is geometrically toward the membrane side. This finding is opposed to previous studies which suggest the reaction zone is closer to the current collector.[22, 42] In the higher performance cells used here different carbon electrode properties and the higher vanadium concentration electrolyte fed at $100 \%$ SoC in a single pass manner facilitate the mass transport of the active species and therefore move the reaction zone.

\subsubsection{Ionic resistivity in the electrode}

We next include the calculated ionic conduction length within the electrode to the NARFB system and estimate the performance. Figure 6 shows the calculation accounting for the losses due to ionic conduction in the separator and in the electrode. In this calculation, electrolyte conductivities at $10^{-2} \mathrm{~S} / \mathrm{cm}$ and $10^{-3} \mathrm{~S} / \mathrm{cm}$ were considered, and rest of the parameters were adopted from Table 1. A significant difference in terms of performance between the VRFB and NARFB arises from the electrolyte conductivity in the electrode. The ionic conduction within the VRFB electrode is mediated by the concentrated sulfuric acid with a conductivity $(0.81 \mathrm{~S} / \mathrm{cm})[43]$ which is higher than that of the non-aqueous electrolyte $\left(10^{-2} \mathrm{~S} / \mathrm{cm}\right)$ by a factor of 80 . The high conductivity of the aqueous electrolyte allows the use of a relatively thick electrode to enhance the surface area without severely sacrificing the performance. The performance of the NARFB system is affected strongly by the length of the ionic pathways. 

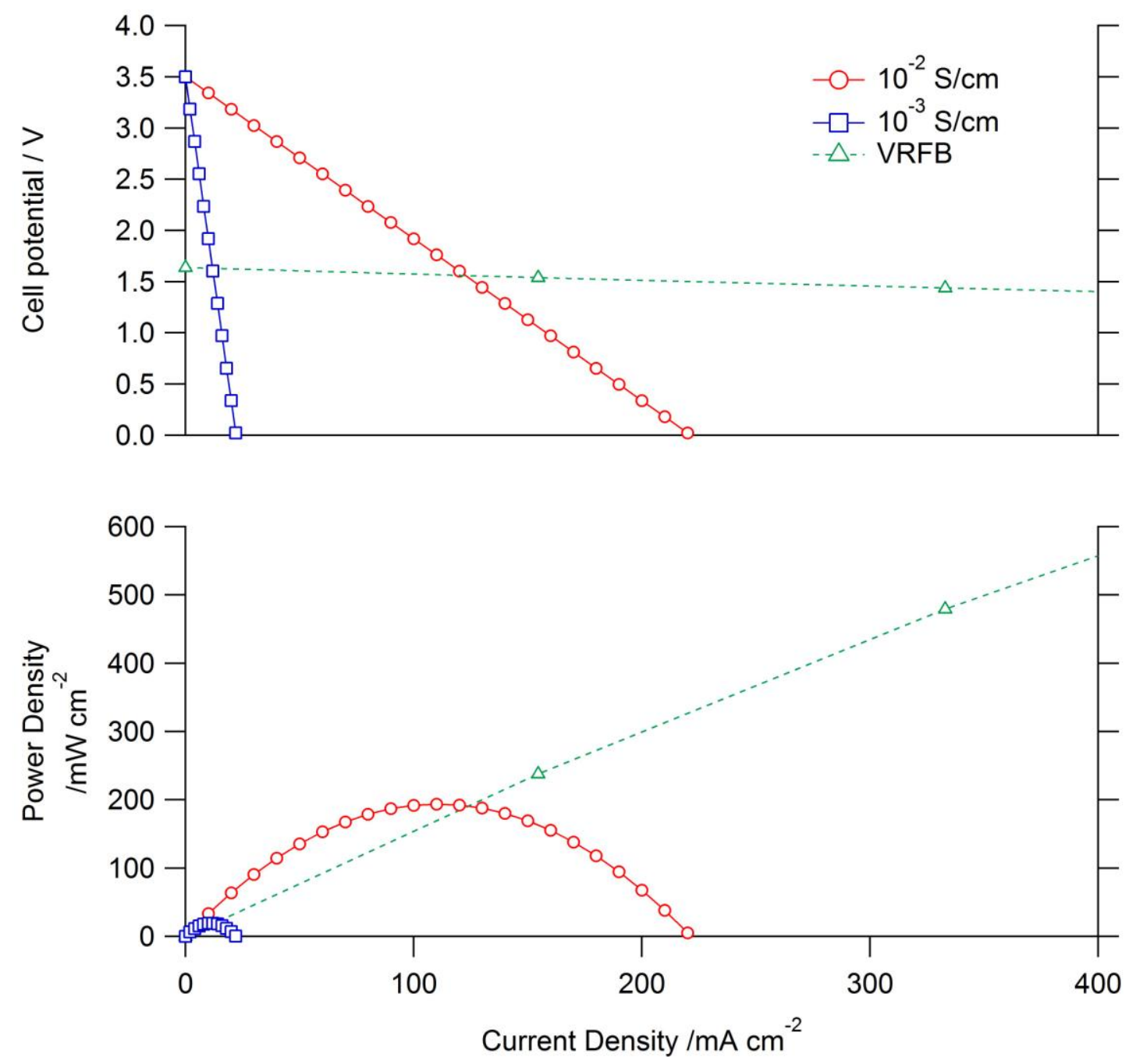

Figure 6. Polarization and power density curves were illustrated by considering the loss due to the electrolyte and the ionic conduction in the electrode. VRFB experimental (dash line) and NARFB calculated with conductivities at $10^{-2} \mathrm{~S} / \mathrm{cm}$ (circles) and $10^{-3} \mathrm{~S} / \mathrm{cm}$ (squares).

\subsubsection{Assuming that we can shorten the length of the ionic pathway}

To decrease ohmic overpotential in the NARFB system, if it is difficult to further increase the electrolyte conductivity, one of the possibilities is to consider a thinelectrode design. Here, we made simple performance estimations of the two systems regarding the ionic conduction length influence within the electrode assuming advances in the NA electrode design can reduce the kinetic losses to insignificant levels as we have shown in the VRFB system. 
Based on the $10^{-2} \mathrm{~S} / \mathrm{cm}$ case in Figure 6, we calculated the NARFB performance with electrode thickness reduced by an order of magnitude. The calculated results are indicated by the solid markers in Figure 7. The performance estimation for VRFB under the same condition is also included for comparison. As the electrode thickness decreases, the performance of the NARFB increases significantly, due to the decreased ohmic loss within the electrodes. The VRB performance calculated with the same conduction length increases as well, but was not as pronounced as the NARFB. As shown in Figure 7, the power density of the NARFB shows an advantage up to $500 \mathrm{~mA} / \mathrm{cm}^{2}$ as compared to the VRFB system, indicating the electrode design is crucial to make NARFB a successful candidate for large scale energy storage application. However, from an application point of view, system operation at high efficiency region is preferred for grid-level applications. In Figure 7, the operating efficiency, defined by the ratio of operating voltage to the OCV. The corresponding current and power densities at $90 \%$ and $80 \%$ operating efficiency for both systems are summarized in Table 2. Note the performance of the VRFB with "thick" electrode is higher than that for the NARFB with "thin" electrode at a given efficiency. The trade-off between the voltage window, the resistance and the performance is clearly indicated in this comparison. 


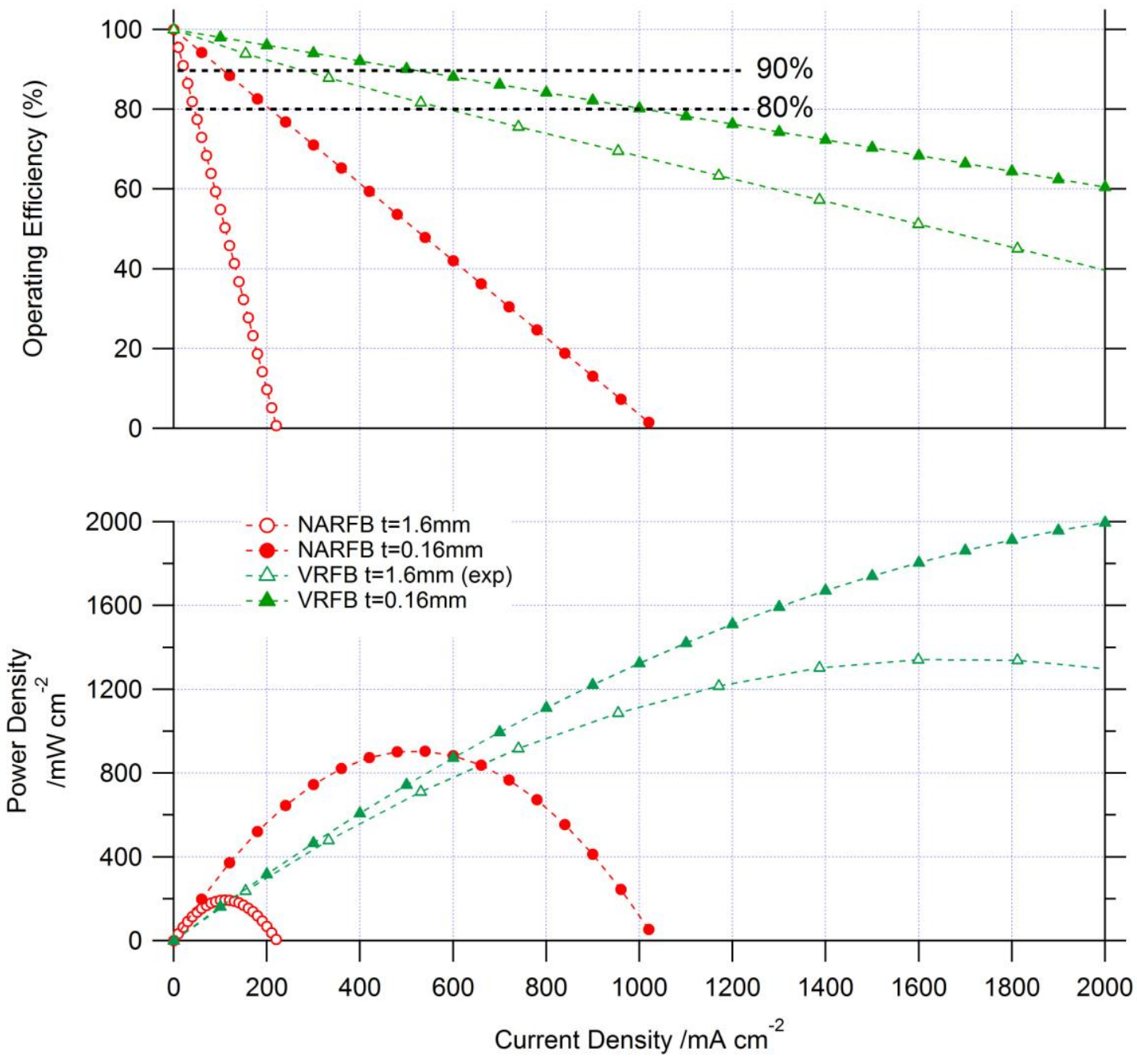

Figure 7. Operating efficiency and power density curves were illustrated by considering the loss due to the electrolyte and the ionic conduction in the electrode along with conduction length effect within the electrode. VRFB (triangles) and NARFB calculated (circles). Solid markers represent the calculated case in which the electrode thickness (t) is $1 / 10$ of the open mark ones.

Table 2. Current density and power density of VRFB and NARFB with different electrode thickness at operating efficiency $90 \%$ and $80 \%$ 


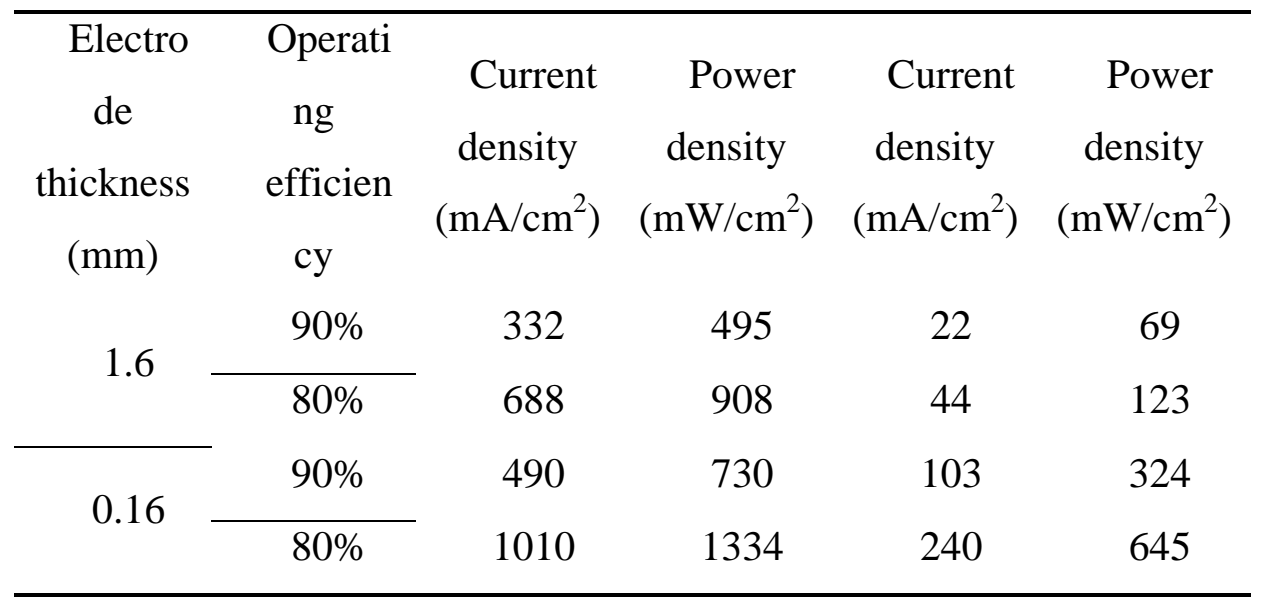

\subsubsection{Evaluation and Extension of Results}

Evidently, the calculated performance for these ohmically-dominated cases reveal a limited but still substantial operating range over which the high open circuit voltage possible in an NARFB leads to higher current density at high voltages. In a battery operating at high efficiency, this is precisely the desired operating range. However, we also see that demanding $80 \%$ or $90 \%$ voltage efficiency significantly narrows the range over which this advantage can be exploited. We also note that many of the factors listed below could reduce peak power density of any flow battery in a practical situation.

Of course, all of the calculations presented above ignore some very important aspects of RFB operation. We now consider the possible impact of what is left out of our estimates. In most of these cases, the situation encountered (performance loss or other aspect) with NARFBs is significantly worse than for the VRFB. This is especially important when considering the grid-scale application of flow batteries.

\subsection{Kinetics}

The calculations presented above disregard the influence of electrode kinetics. For the VRFB, we find that suitable electrodes with sufficient, accessible surface area substantially eliminate this source of loss. Because the NARFB must operate in a low current range, it is likely that the performance of this type of flow battery will be more sensitive to any kinetic losses.

\subsection{Available materials choices}


While we have focused primarily on the active cell components in our analysis, the use of an open flowing system puts additional constraints on the non-aqueous system in several respects. Material compatibility issues are generally more severe for such systems. Appropriate seals and gaskets are already major practical issues in these systems and the use of non-aqueous solvents is likely to significantly circumscribe the available solutions. This is also true for pumps used in the system.

\subsection{Shunt currents}

Most approaches to minimizing the influence of shunt currents, currents that flow external to the cell because of the continuous electrolyte path connecting electronically conducting elements of different voltages, focus on increasing the resistance of the path to the cell inlet. The higher resistance of non-aqueous fluids may have a beneficial effect in this regard.

\subsection{Cost}

To have any chance of being deployed on the grid, low cost of the system is essential. Few solutions of any type could compete with aqueous sulfuric or hydrochloric acid for low cost. Non-aqueous solvents will be significantly more expensive, as will the salts used in the electrolyte. A common approach used to achieve high solubility and the widest possible voltage separation in non-aqueous systems is the deployment of complexing agents. Unless abundant compounds are used for this, it is highly likely that substantial cost disadvantage will accrue to the non-aqueous system. Finally, from the analysis presented above, to achieve efficiency targets the NARFB will operate at relatively low current density, even in a favorable scenario of high voltage and at the high end of the range of conductivity. This entails use of a much higher surface area in the cell, with cost accruing to each component. In short, the potential for low installed cost in a large-scale is much lower for NARFBs.

\subsection{Safety}

Generally, non-aqueous solvents that have suitable characteristics for use in this application, such as relatively low viscosity and high conductivity, will be rather flammable. This is certainly so when compared to the aqueous solutions used in VRFBs.

\subsection{Intended application}


Here we have focused on the promising application of RFBs for Grid-Scale energy storage. Based on our analysis of achievable performance using very generous, best-case parameters, the profitable deployment of NARFBs for this application is somewhat problematic even if one ignores the immediately preceding litany of issues. However, there may be 'mission-critical' applications where the intrinsic high energy density possible from some systems can overcome these considerations. This is possible at smaller scale.

\section{Conclusions}

In this work, we report a high performance all-vanadium redox flow battery with improved solution, electrodes and membrane. The polarization curve exhibits a straight line, suggesting that the ohmic process is dominating the loss. The IR-free voltage also shows a linear behavior as a function of current density. We therefore attribute the IRfree loss to the ionic resistance within the electrode. The performance of idealized nonaqueous redox flow batteries, estimated by considering the ohmic loss only, is more limited by the ohmic characteristics of the electrolyte. This limiting performance decreases even more dramatically when the ohmic loss within the electrode was included. Indeed, the substantial advantage of NARFBs, the potential for high energy density due to a high voltage window and appropriate redox couples, does not necessarily lead to high performance over a substantial current density window. Thin electrodes with sufficient surface area to retain the minimal kinetic loss are needed for high performance non-aqueous systems. Even in this case, however, a plethora of other disadvantageous criteria render the NARFB a technology that is must be looked at with some skepticism within the realm of presently known materials and their costs and safety implications. While there may be applications where the energy density achieved with the NARFB outweighs all other aspects, these are not yet evident. It is also possible that significant improvement can be made in the overall electrolyte conductivity and in the characteristics

of materials discussed here. These will be necessary to make the NARFB competitive in terms of power or current density.

\section{Acknowledgements}


The authors gratefully acknowledge the support of the US Department of Energy Office of Electricity Delivery and Reliability Program, managed by Dr. Imre Gyuk, and the U. S. Office of Naval Research for support of this work.

\section{References}

1. J.P. Holdren, Science (New York, N.Y.), 2007, 315, 737.

2. V.S. Arunachalam and E.L. Fleischer, MRS Bull., 2011, 33, 264.

3. D. Ginley, M.A. Green and R. Collins, MRS Bull., 2011, 33, 355.

4. Z. Yang, J. Zhang, M.C.W. Kintner-Meyer, X. Lu, D. Choi, J.P. Lemmon and J. Liu, Chem. Rev., 2011, 111, 3577.

5. C. Ponce de León, A. Frías-Ferrer, J. González-García, D.A. Szánto, F.C. Walsh, J. Power Sources, 2006, 160, 716.

6. A.Z. Weber, M.M. Mench, J.P. Meyers, P.N. Ross, J.T. Gostick and Q. Liu, J. Appl. Electrochem., 2011, 41, 1137.

7. M. Skyllas-Kazacos, M.H. Chakrabarti, S.A. Hajimolana, F.S. Mjalli, M. Saleem, J. Electrochem. Soc., 158, (2011), R55-R79.

8. P.K. Leung, X. Li, C. Ponce de Léon, L. Berlouis, C.T.J. Low, F.C. Walsh, RSC Advances, 2 (27), (2012), 10125 - 10156.

9. G. Kear, A.A. Shah, F.C. Walsh, Int. J. Energy, 36, (2011), 1105-1120.

10. G. Codina and A. Aldaz, J. Appl. Electrochem., 1992, 22, 668.

11. M. Skyllas-Kazacos, J. Electrochem. Soc., 1986, 133, 1057.

12. E. Sum and M. Skyllas-Kazacos, J. Power Sources, 1985, 15, 179.

13. A. Price, S. Bartley and S. Male, Power Eng. J., 1999, 13, 122.

14. A.E.S. Sleightholme, A.A. Shinkle, Q. Liu, Y. Li, C.W. Monroe and L.T. Thompson, J. Power Sources, 2011, 196, 5742.

15. Q. Liu, A.E.S. Sleightholme, A.A. Shinkle, Y. Li and L.T. Thompson, Electrochem. Commun., 2009, 11, 2312.

16. W. Wang, W. Xu, L. Cosimbescu, D. Choi, L. Li and Z. Yang, Chem. Commun., 2012, 48, 6669.

17. Y. Lu and J.B. Goodenough, J. Mater. Chem., 2011, 21, 10113. 
18. M. Zhang, M. Moore, J.S. Watson, T.A. Zawodzinski and R.M. Counce, J. Electrochem. Soc., 2012 , 159, A1183.

19. M. Moore, J. Watson, T.A. Zawodzinski Jr., M. Zhang and R.M. Counce, ECS Trans., 2012. 41, 1.

20. K.T. Cho, P. Ridgway, A.Z. Weber, S. Haussener, V. Battaglia and V. Srinivasan, J. Electrochem. Soc., 2012, 159, A1806.

21. D.S. Aaron, Q. Liu, Z. Tang, G.M. Grim, A.B. Papandrew, A. Turhan, T.A. Zawodzinski and M.M. Mench, J. Power Sources, 2012, 206, 450.

22. Q.H. Liu, G.M. Grim, A.B. Papandrew, A. Turhan, T.A. Zawodzinski and M.M. Mench, J. Electrochem. Soc., 2012, 159, A1246.

23. S. Hamelet, T. Tzedakis, J.-B. Leriche, S. Sailler, D. Larcher, P.-L. Taberna, P. Simon and J.-M. Tarascon, J. Electrochem. Soc., 2012, 159, A1360.

24. R.. Darling, K. Gallagher, J. Kowalski, S. Ha and F. Brushett, Environ. Sci., 2014, 7, 3459.

25. K. Xu, Chem. Rev., 2004, 104, 4303.

26. S.S. Zhang, J. Power Sources, 2007, 164, 351.

27. P. Arora and Z.J. Zhang, Chem. Rev., 2004, 104, 4419.

28. A. Manuelstephan, Eur. Polym. J., 2005, 42, 21.

29. F.M. Gray, Solid Polymer Electrolytes: Fundamentals and Technological Applications, John Wiley \& Sons, 1991.

30. S.-H. Shin, S.-H. Yun and S.-H. Moon, RSC Adv., 2013, 3, 9095.

31. J. Fu, Solid State Ionics, 1997, 96, 195.

32. J.B. Bates, N.J. Dudney, G.R. Gruzalski, R.A. Zuhr and A. Choudhury, J. Power Sources, 1993, 44, 103.

33. A.A. Shinkle, A.E.S. Sleightholme, L.D. Griffith, L.T. Thompson and C.W. Monroe, J. Power Sources, 2012, 206, 490.

34. A.A. Shinkle, A.E.S. Sleightholme, L.T. Thompson and C.W. Monroe, J. Appl. Electrochem., 2011, 41, 1191.

35. J.-H. Kim, K.J. Kim, M.-S. Park, N.J. Lee, U. Hwang, H. Kim and Y.-J. Kim, Electrochem. Commun., 2011, 13, 997. 
36. T. Yamamura, Y. Shiokawa, H. Yamana, H. Moriyama, Electrochim. Acta, 2002, 48, 43.

37. Y. Matsuda, K. Tanaka, M. Okada, Y. Takasu, M. Morita and T. Matsumura-Inoue, J. Appl. Electrochem., 1988, 18, 909.

38. J. Mun, M.-J. Lee, J.-W. Park, D.-J. Oh, D.-Y. Lee and S.-G. Doo, Electrochem. Solid-State Lett., 2012, 15, A80.

39. M.H. Chakrabarti, R.A.W. Dryfe and E.P.L. Roberts, Electrochim. Acta, 2007, 52, 2189.

40. M.H. Chakrabarti, E.P.L. Roberts, C. Bae and M. Saleem, Energy Convers. Manage., 2011, 52, 2501.

41. D. Zhang, Q. Liu, X. Shi and Y. Li, J. Power Sources, 2012, 203, 201.

42. G. Qiu, A.S. Joshi, C.R. Dennison, K.W. Knehr, E.C. Kumbur, Y. Sun, Electrochim. Acta, 2012, 64, 46.

43. H.E. Darling, J. Chem. Eng. Data, 1964, 9, 421-426. 